\title{
Clinical outcomes among hospital patients with Middle East respiratory syndrome coronavirus (MERS-CoV) infection
}

\author{
Abdulrahman Mohammed G. Habib', Mohamed Abd Elghafour Ali', Baha R. Zouaoui ${ }^{1}$, Mustafa Ahmed H. Taha', \\ Bassem Sahsah Mohammed ${ }^{2}$ and Nazmus Saquib ${ }^{1 *}$ (i)
}

\begin{abstract}
Background: Mortality is high among patients with Middle East Respiratory Syndrome Coronavirus (MERS-CoV) infection. We aimed to determine hospital mortality and the factors associated with it in a cohort of MERS-CoV patients.

Methods: We reviewed hospital records of confirmed cases (detection of virus by polymerase chain reaction from respiratory tract samples) of MERS-CoV patients $(n=63)$ admitted to Buraidah Central Hospital in Al-Qassim, Saudi Arabia between 2014 and 2017. We abstracted data on demography, vital signs, associated conditions presented on admission, pre-existing chronic diseases, treatment, and vital status. Bi-variate comparisons and multiple logistic regressions were the choice of data analyses.

Results: The mean age was 60 years (SD = 18.2); most patients were male (74.6\%) and Saudi citizens (81\%). All but two patients were treated with Ribavirin plus Interferon. Hospital mortality was $25.4 \%$. Patients who were admitted with septic shock and/or organ failure were significantly more likely to die than patients who were admitted with pneumonia and/or acute respiratory distress syndrome $(\mathrm{OR}=47.9,95 \% \mathrm{Cl}=3.9,585.5, p$-value 0.002). Age, sex, and presence of chronic conditions were not significantly associated with mortality.
\end{abstract}

Conclusion: Hospital mortality was $25 \%$; septic shock/organ failure at admittance was a significant predictor of mortality.

Keywords: Ribavirin, Interferon alpha, MERS-CoV, Mortality

\section{Background}

The Middle East Respiratory Syndrome Coronavirus (MERS-CoV) infection is a recent and fatal disease, detected first in Saudi Arabia, where the majority of cases have so far occurred. Subsequently, it spread through the Arabian Peninsula and into neighbouring Middle Eastern countries before it became a global concern, reaching as far as the Korean Peninsula. By February 2018, its presence was detected in 27 countries worldwide, with 2144 recorded cases, out of which 750 resulted in death [1]. MERS-CoV is a contagious disease caused by $\mathrm{C}$ lineage of $\beta$-coronavirus. The infection can

\footnotetext{
* Correspondence: a.saquib@sr.edu.sa

${ }^{1}$ College of Medicine, Sulaiman Al Rajhi Colleges, P.O. Box 777, Bukayriah,

Al-Qassim Zip code 51941, Saudi Arabia

Full list of author information is available at the end of the article
}

occur either through exposure with an infected animal or human [2]. Dromedary camels are believed to have been the carrier of MERS-CoV for decades as camels of the Middle Eastern region appear to be the only zoonotic host able to transmit infection to humans [3]. There is evidence of super spreading of infection (i.e., a single patient infects a disproportionate number of contacts) in MERS-CoV, and therefore, healthcare workers who provide support to infected patients are particularly vulnerable [4]. The disease exhibits a wide range of presentations at diagnosis, e.g., from no symptoms to subtle signs of pneumonia to multi-organ failure, and has the capacity to progress rapidly to cause death $[5,6]$. At present, there is no effective vaccine available to prevent this fatal infection [1].

(c) The Author(s). 2019 Open Access This article is distributed under the terms of the Creative Commons Attribution 4.0 International License (http://creativecommons.org/licenses/by/4.0/), which permits unrestricted use, distribution, and 
There have been both animal and human studies on treatment efficacy for MERS-CoV infection. A common form of treatment is antiviral drugs that target specific parts of the $S$ protein in MERS-CoV. These are known as anti-MERS-CoV neutralizing monoclonal antibodies (mAbs), anti-dipeptidyl peptidase 4 (DPP4) mAbs, peptidic fusion inhibitors, siRNA, and others [7]. MERSCoV binds with DPP4, which is found on the surface of cells in the lungs and kidneys. Protein-targeting mAbs in mice were not reported to have given in vivo protection from MERS-CoV; nevertheless, mAbs variants, including mersmab1, 2E6 and 4C2, were found to prevent entry into DPP4 cells and effectively neutralise live MERS$\mathrm{CoV}$ infection in mice $[8,9]$. DPP4 antagonists target the receptor-binding domain (RBD), competing with and inhibiting MERS-CoV infection. The DPP4 antagonists used in ferrets were found to be highly protective against MERS-CoV entry [10]. Multiple RBD-mAbs were found to elicit protective and therapeutic abilities against MERS-CoV infectivity in humanised DPP4 mice and other variants, as well as in rhesus monkeys $[8,11-13]$.

The drugs that have been tested in humans included Interferon (alpha and beta), antiviral nucleoside analogues (Ribavirin), serine protease inhibitors (Camostat), immunosuppressant (cyclosporine, mycophenolate mofetil), monoclonal antibodies, and broad-spectrum antivirals (Nitazoxanide) [14]. In one study, multiple regimens were tested including mycophenolate mofetil, Interferon alpha and beta with or without ribavirin combination, and hydrocortisone [15]. Similarly, the efficacy of Interferon-beta with lopinavir-ritonavir has been the focus of an on-going clinical trial [16]. The most widely tested regimen, however, has been Ribavirin in combination with Interferon. This regimen has been found effective in reducing the virus replication 'in vitro' [17]. It has also modulated the host response and improved the clinical outcome in animal experiments [18].

Clinical outcomes of MERS-Cov patients varied substantially among previous studies. For example, hospital mortality was as low as $4 \%$ in one study [19] but as high as $100 \%$ in another study [20]. In a majority of studies, the mortality ranged between 20 and $60 \%$ [6, 21-28]. Small sample sizes $(5,14,20$, and 31$)$ in some of the included studies likely contributed in the variation of the morality estimate $[6,20,21,29]$. Additionally, the mortality rate was influenced by the patients' demography (e.g., age), physical conditions at admission (e.g., fever, shock, organ failure etc.), pre-existing diseases such diabetes or hypertension, or choice of treatment $[26,30]$.

We, therefore, present data of a larger clinical study $(n=63)$ of confirmed cases of MERS-CoV patients who were admitted to a referral hospital in the Al-Qassim region of Saudi Arabia between 2014 and 2017. Most of these patients received Ribavirin plus Interferon alpha as treatment. We ascertained their hospital mortality and assessed whether it varied by age, sex, or pre-existing comorbid conditions.

\section{Methods}

We used the medical records of Buraidah Central Hospital $(\mathrm{BCH})$ in the $\mathrm{Al}$-Qassim region and adopted a retrospective cohort design for this study. $\mathrm{BCH}$ is the main centre in the region for the treatment of MERS$\mathrm{CoV}$ infections; patients are referred here from neighbouring secondary hospitals (e.g., King Saud Hospital in Unaizah, Sulaiman Al Habib in Buraidah, and hospitals in ArRass, AlMuthnab, and Bukayriah) and tertiary hospitals (e.g., King Fahad Specialist hospital and Prince Sultan Cancer Centre in Buraidah).

The study inclusion criteria were: (1) adult (above 17 years), (2) laboratory-confirmed MERS-CoV infection with PCR (polymerase chain reaction) detection of the virus in samples taken from the respiratory tract of the patient, and (3) patients admitted to BCH between 2014 and 2017. The exclusion criteria were: (1) suspected cases of MERS-CoV without a confirmed diagnosis, and (2) pregnant women.

We evaluated $\approx 1000$ medical records of suspected cases of MERS-CoV and excluded the ones that did not meet the eligibility criteria. The majority did not have laboratory confirmation of a MERS-CoV infection. The accuracy of data for any patient referred to $\mathrm{BCH}$ was crosschecked with the patient's record from the original hospital. A total of 63 patients met the eligibility criteria and are the focus of this report. Out of 63, 43 patients' data came from the Department of Archives at $\mathrm{BCH}$; the remaining 20 came from the Department of Infection Control (from discharge summaries) at $\mathrm{BCH}$ since they had not yet been archived (Fig. 1). Some key information (temperature, heart rate, blood pressure, and respiratory rate) was missing from patients' records at Infection Control. All acquired data were crosschecked with their respective patient file numbers by two co-authors to ensure there was no data duplication.

The exposure variable was the treatment regimen of $\mathrm{Ri}$ bavirin plus Interferon alpha, which was the treatment of choice at $\mathrm{BCH}$. The outcome variable was mortality during hospital stay. The covariates were age (in years), sex (male, female), nationality (Saudi, Non-Saudi), admission year (2014-2015, 2016-2017), vital signs on admission (body temperature, heart rate, respiratory rate, systolic and diastolic blood pressure), MERS-CoV associated conditions on admission (pneumonia/acute respiratory distress syndrome, septic shock/multiple organ failure, abdominal pain/diarrhoea), pre-existing comorbid conditions on admission (diabetes, hypertension, hepatitis $\mathrm{C}$, chronic renal failure, and chronic heart disease), receipt of the treatment regimen (yes, no), duration of hospital stay (in days), status at discharge (alive, dead). 


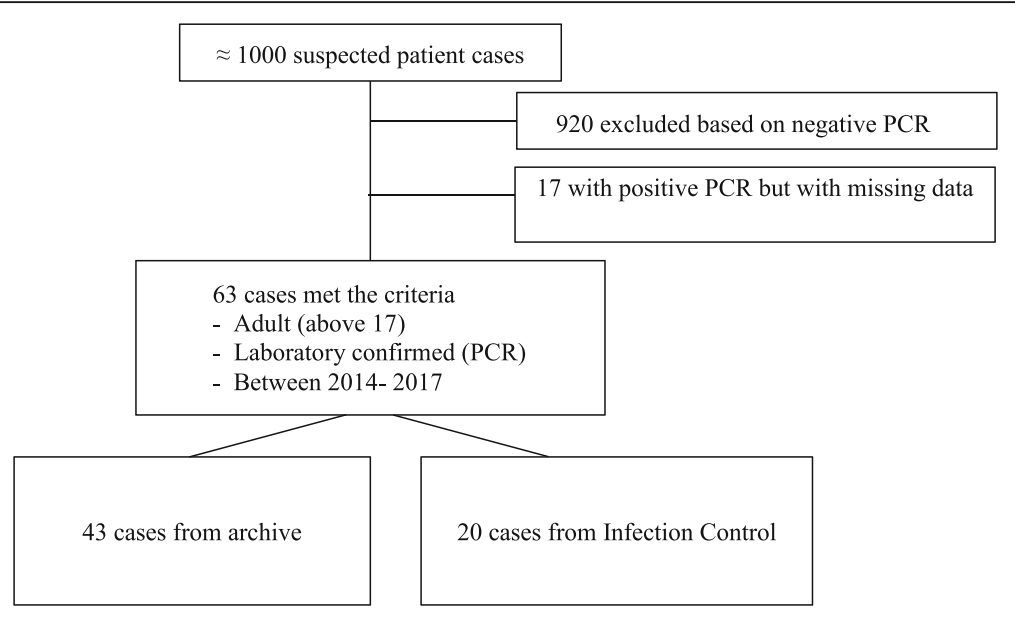

Fig. 1 Methods

The Regional Ethical Committee of Al-Qassim and the administration of the $\mathrm{BCH}$ approved this study. The ethical committee did not require that we obtain informed consent from the patients since we examined archived medical records, had no direct contact with the patients, and ensured that the data collectors abstracted and recorded the patient data anonymously.

\section{Analyses}

We entered and analysed the data with SPSS (version 23 ); we used a two-sided test with an alpha of 0.5. We calculated mean and standard deviation for continuous variables and frequency for categorical variables. From the comorbid conditions on admission, we created a summary variable with zero, one, and two or more conditions as levels. We calculated hospital mortality as the number of dead patients over the total number of patients admitted with a MERS-CoV diagnosis. We compared the hospital mortality between patients who did and did not receive the combination therapy. The observed mortality difference, the $p$-value of the test, and the sample size were used in the power calculation (power $=69 \%$ ). In addition, we compared demographics, vital signs, MERS-CoV-associated conditions on admission, the number of comorbid conditions on admission, and duration of hospital stay between those who were alive and those who were dead at hospital discharge. We used Chi-square and t-test respectively for comparison of categorical and continuous variables. Finally, we used a binary multiple logistic regression model to identify correlates of hospital mortality (yes, no). We considered age, sex, admission status, and number of chronic diseases for inclusion in the model. For model robustness and ease of interpretation, we made the number of chronic diseases variable binary (no, yes). Similarly, we added the one patient who presented with abdominal pain and diarrhoea to the group with pneumonia and ARDS. We expressed the associations with odds ratio and their associated 95\% confidence interval and checked model adequacy with Hosmer-Lemeshow Goodness of fit statistic.

\section{Results}

Table 1 compares patients' vital status by age, length of hospital stay, and vital signs on the day of admission. The sample's mean (standard deviation) age was 59.7 (18.2) years. The majority of the patients were male (74.6\%, $n=47)$ and Saudi citizens $(81 \%, n=51)$; the largest number of patients was admitted between 2016 and $2017(n=52)$ (data not shown). Hospital mortality was $25.4 \%$ (16/63); mortality among patients who received the combination therapy was $22.9 \%(14 / 61)$. Mean age did not differ (58.3 vs. 63.9 years; $p$-value $=0.542)$ between those who survived and those who died. Duration of hospital stay was significantly shorter for patients who survived (8.7 vs. 17.4 days; $p$-value $<0.0001)$. Mean heart

Table 1 Comparison of vital status by age, duration of hospital stay, and vital signs on admission in a sample of MERS-CoV patients $(n=63)$

\begin{tabular}{lllll}
\hline Variables & $N$ & Vital Status & P-value \\
\cline { 3 - 4 } & & $\begin{array}{l}\text { Alive }(n=47) \\
\text { Mean (S.D.) }\end{array}$ & $\begin{array}{l}\text { Dead }(n=16) \\
\text { Mean (S.D.) }\end{array}$ \\
\hline Age $^{\mathrm{a}}$ & 63 & $58.3(18.4)$ & $63.9(17.5)$ & 0.542 \\
Duration of stay in days & 43 & $8.7(4.3)$ & $17.4(21.9)$ & $<0.001$ \\
Temperature $^{\circ} \mathrm{C}$ & 43 & $37.0(1.3)$ & $37.8(0.9)$ & 0.992 \\
Heart rate & 43 & $85.5(12.1)$ & $110.1(24.6)$ & 0.015 \\
Respiratory rate & 43 & $22.1(3.8)$ & $22.8(7.9)$ & 0.004 \\
Systolic blood pressure & 43 & $127.2(19.9)$ & $128.5(42.1)$ & 0.001 \\
Diastolic blood pressure & 43 & $70.2(9.6)$ & $63.8(22.1)$ & 0.001 \\
\hline
\end{tabular}

available for all patients 
rate ( 85.5 vs. 110.1 beats; $\mathrm{p}$-value $=0.015)$, respiratory rate $(22.1$ vs. 22.8 breaths per minute; $p$-value $=0.004)$, and systolic blood pressure (127.2 vs. $128.5 \mathrm{mmHg}$; $p$ value $=0.001$ ) were significantly lower among those who survived; on the other hand, their mean diastolic blood pressure was significantly higher (70.2 vs. 63.8 mmHg; $p$-value $=0.001$ ) (comparison dead patients). Body temperature on admission did not differ between the groups $\left(37\right.$ vs. $37.8^{\circ} \mathrm{C}$; $p$-value $\left.=0.99\right)$.

Table 2 compares patients' vital status in relation to their admission status, number of diseases on hospital arrival, and intake of combination regimen. Pneumonia was the most common presenting manifestation $(n=55$, $87.3 \%)$, followed by septicaemia $(n=07 ; 11 \%)$ and abdominal pain/diarrhoea $(n=1 ; 2 \%)$. Patients who survived were more likely to have had pneumonia (95\% vs. $62.5 \%)$ and less likely to have had septic shock ( $2.1 \%$ vs. $37.5 \%)$ at admission than patients who died ( $p$-value $<0.0001)$. Thirtyeight percent $(38 \%)$ of patients had no comorbid conditions, $14 \%$ had one condition, and $48 \%$ had two or more conditions. Patients who survived were less likely to have two or more chronic conditions than patients who died ( $42.6 \%$ vs. $62.5 \%)$, but the difference was not statistically significant $(p$-value $=0.13$ ). All but two MERS-CoV patients received combination therapy of Ribavirin and Interferon $(97 \%$; 61/63). Patients who survived were more likely to have had received the combination therapy than patients who died $(100 \%$ vs. $87.5 \%$; p-value $=0.01)$.

The odds of hospital mortality increased if the MERS$\mathrm{CoV}$ patients were older or female. For example, females were 2.4 times more likely to die compared to males. On the other hand, the odds of death were lower $(\mathrm{OR}=$

Table 2 Comparison of vital status by admission status, number of diseases at admission, and treatment in a sample of MERSCoV patients $(n=63)$

\begin{tabular}{|c|c|c|c|c|}
\hline \multirow[t]{2}{*}{ Admission Status } & \multirow[t]{2}{*}{$N$} & \multicolumn{2}{|l|}{ Vital Status } & \multirow[t]{2}{*}{$P$-value } \\
\hline & & Alive N (\%) & Dead N (\%) & \\
\hline Pneumonia / ARDS ${ }^{a}$ & 55 & $45(95.7)$ & $10(62.5)$ & $<0.0001$ \\
\hline Septic Shock / MOF ${ }^{b}$ & 7 & $1(2.1)$ & $6(37.5)$ & \\
\hline Abdominal pain and diarrhoea & 1 & $1(2.1)$ & $0(0.0)$ & \\
\hline \multicolumn{5}{|l|}{ N. of diseases at admission ${ }^{c}$} \\
\hline No chronic disease & 24 & $18(38.3)$ & $6(37.5)$ & 0.131 \\
\hline One chronic disease & 9 & $9(19.1)$ & $0(0.0)$ & \\
\hline Two or more diseases & 30 & $20(42.6)$ & $10(62.5)$ & \\
\hline \multicolumn{5}{|l|}{ Management } \\
\hline Took the regimen $^{d}$ & 61 & $47(100.0)$ & $14(87.5)$ & 0.014 \\
\hline Did not take the regimen & 2 & $0(0.0)$ & $2(12.5)$ & \\
\hline
\end{tabular}

${ }^{\mathrm{a} A R D S}$ : Acute respiratory distress syndrome

${ }^{\mathrm{b}}$ MOF: Multiple organ failure

${ }^{\mathrm{C}}$ Chronic diseases included diabetes, hypertension, hepatitis $\mathrm{C}$, chronic renal

diseases, and chronic heart diseases

${ }^{\mathrm{d}}$ All regimens included the combination therapy of Interferon alpha and Ribavirin
0.21 ) in patients with at least one chronic condition (reference $=$ no chronic diseases). None of these associations, however, were statistically significant $(p>0.05)$. The only significant association was if patients were admitted to the hospitals with shock or organ failure $(\mathrm{OR}=$ 47.9) $($ reference $=$ pneumonia $/$ ARDS $)($ Table 3$)$.

\section{Discussion}

Overall, 25\% of the patients died from MERS-CoV infection in our study group. Pneumonia and acute respiratory distress syndrome were the most common associated conditions that MERS-CoV patients had when they were admitted to the hospital. The patients who died had worse vital signs (such as heart and respiratory rate) at admission than patients who survived. Age, sex, or number of chronic conditions was not significantly associated with hospital mortality. Presenting with septic shock and organ failure at admission was significantly associated with hospital mortality. This was a novel finding, but the odds ratio that we reported for septic shock/organ failure should be interpreted with caution due to a very small number of patients with that condition $(n=07)$.

Three local studies reported a hospital mortality of between 22 and 28\%, and an international study reported $20.4 \%$ mortality among MERS-CoV patients [19, 29-31], which is very similar to what we found in our study (25\%). The similarity in mortality between these studies could be due to the aggressive and focused identification of suspected cases and dealing with them properly and early according to protocol. It should be noted that the mortality rate of our study was much lower than what other studies had reported (range 35-60\%) [6, 20-28].

Our reported hospital mortality of patients who received combination therapy of Ribavirin and Interferon (22.9\%) was also comparable to that of a study by Omrani et al., who reported $30 \%$ mortality among 20 patients who received the same combination therapy [29]. This mortality rate was much lower than what was reported by $\mathrm{Al}$ Mekhlafi et al. $(n=31.39 .1 \%)$, Khaild et al. $(n=11,55 \%)$, and Al-Tawfiq et al. $(n=5,100 \%)[6,20,21]$. The mortality with this combination therapy was found to be only $4 \%$ $(1 / 24)$ in a Korean study [19]. Choi et al. attributed this reduced mortality in Koreans to the aggressive antiviral therapy, mechanical ventilatory support, and extracorporeal membrane oxygenation [19].

The variability of mortality between the studies on Ribavirin plus Interferon can be partly explained by the variability of patients' age distribution, their physical condition, and the complications of MERS-CoV that they had at the time of admission. For example, our patients were relatively younger than patients of those studies that reported higher mortality $[26,27]$, and older than studies that reported a lower mortality [19]. Multiple studies found mortality increased substantially with age $[26-28,30]$. 
Table 3 Adjusted associations of hospital mortality in a sample of MERS-CoV patients $(n=63)$

\begin{tabular}{|c|c|c|c|c|c|}
\hline Variable & Level & $\mathrm{N}$ & OR & $95 \% \mathrm{Cl}$ & $P$-value \\
\hline Age & (in years) & 63 & 1.03 & $0.99-1.07$ & 0.19 \\
\hline \multirow[t]{2}{*}{ Sex } & Male & 47 & 1.0 & & \\
\hline & Female & 16 & 2.4 & $0.49-11.6$ & 0.28 \\
\hline \multirow[t]{2}{*}{ Admission status } & Pneumonia / ARDS & 56 & 1.0 & & \\
\hline & Septic Shock / MOF ${ }^{b}$ & 07 & 47.9 & $3.9-585.5$ & 0.002 \\
\hline \multirow[t]{2}{*}{ N. of diseases at admission ${ }^{c}$} & None & 24 & 1.0 & & \\
\hline & At least one & 39 & 2.1 & $0.04-1.13$ & 0.07 \\
\hline
\end{tabular}

${ }^{a}$ ARDS: acute respiratory distress syndrome

${ }^{\mathrm{b}}$ MOF: Multiple organ failure

${ }^{c}$ Chronic diseases included diabetes, hypertension, hepatitis C, chronic renal diseases, and chronic heart diseases

Fever was the most common symptom reported in other studies and affected the majority of patients (between 69 and $98 \%$ of patients) [19, 22, 28, 30]. However, a smaller number of patients in our study were admitted with fever (defined as temperature $>37.4^{\circ}=45 \%$ ). This could indicate that our patients came to the hospital at an early stage of infection, or that they used over-thecounter antipyretics before they were brought to the hospital. Finally, the studies that reported a higher mortality with the combination therapy had patients who were seriously ill with the infection; many of them were in the intensive care unit and received respiratory resuscitation by intubation or mechanical ventilation $[6,20]$. Unfortunately, we lacked this critical information about our patients, and therefore, were not able to make a comparison in this respect.

Hypertension (55.6\%) and diabetes (47.6\%) were the most common chronic conditions among our patients; this was the case in other studies as well $[26,30]$. Although the finding was not statistically significant, the direction of the association between the number of chronic conditions and mortality among MERS-CoV patients was similar to that of other studies that reported a higher mortality among those who were diabetic, hypertensive or had chronic kidney disease [26, 30].

Our study had several limitations. Although we included a relatively large number of participants, the size was still very small $(n=63)$. This could possibly explain some of the non-significant associations that we have found (e.g., sex, number of chronic conditions). We had to rely on paper-based patient records for data abstraction; some handwriting was not legible, and it is known that handwritten records are more prone to error than electronic data. In addition, the 20 patient files that were not part of the archive were very brief, and only a certain amount of information from them was usable. We did not follow patients beyond their discharge from the hospitals; hence, we could not comment on their long-term prognosis, which one study did [6]. Unlike a few other studies [21, 22], our patients almost exclusively received treatment with one regimen, i.e., Ribavirin and Interferon. The lack of a reference group (i.e., patients who received other regimens) prevented us from determining the true efficacy of this combination therapy. Finally, our study included patients 35 years or older, and therefore, we are unable to comment on the clinical outcomes in children or adolescents with MERS-CoV infection.

\section{Conclusion}

In our sample of MERS-Cov patients, hospital mortality was $25 \%$. Death was more likely if the patients came to the hospital with septic shock or organ failure. We were unable to compare the efficacy of the combination therapy of Ribavirin plus Interferon on mortality in the absence of patients who received other types of treatment. Future studies should strive for a larger sample, ensure inclusion of patients who received disparate therapies, and collect comprehensive information on the presenting signs and symptoms and co-exiting conditions.

\section{Abbreviations \\ ARDS: Acute respiratory distress syndrome; $\mathrm{BCH}$ : Buraidah Central Hospital; DPP4: Anti-dipeptidyl peptidase 4; mAbs: Anti-MERS-CoV neutralising monoclonal antibodies; MERS-CoV: Middle East Respiratory Syndrome Coronavirus; MOF: Multiple organ failure; RBD: Receptor-binding domain}

\section{Acknowledgments}

The authors thank Dr. Mohamed S. Zaghloul for assistance with data analysis. We are grateful to Ms. Erin Strotheide for her editorial contributions to this manuscript. We are thankful to all physicians, hospital staff and administration at $\mathrm{BCH}$. We thank the BCH Department of Archives for facilitating data collection at their hospital.

\section{Authors' contributions}

AH and NS had the original idea and developed the study protocol. AH, MA, BRZ, and MT coordinated the fieldwork with Buraidah Central Hospital (BCH). BSM was involved with data management. AH performed the analysis and drafted the initial manuscript. All authors contributed in the preparation of manuscript writing, and approved its final version.

\section{Funding}

This work was supported by Sulaiman Al Rajhi Colleges, Saudi Arabia, but it had no role in the design, analysis, preparation of the manuscript, or decision to publish. 


\section{Availability of data and materials}

The datasets used and/or analysed during the current study are available from the corresponding author on reasonable request.

\section{Ethics approval and consent to participate}

The Regional Ethical Committee of Al-Qassim and the administration of the $\mathrm{BCH}$ approved this study. The ethical committee did not require that we obtain informed consent from the patients since we examined archived medical records, had no direct contact with the patients, and ensured that the data collectors abstracted and recorded the patient data anonymously. Administrative permission was sought and obtained from the director of $\mathrm{BCH}$ to access the data.

\section{Consent for publication}

Not applicable.

\section{Competing interests}

The authors declare that we received no support from any organization for the submitted work and that they had no financial relationships with any organization that might have an interest in the submitted work.

\section{Author details}

${ }^{1}$ College of Medicine, Sulaiman Al Rajhi Colleges, P.O. Box 777, Bukayriah, Al-Qassim Zip code 51941, Saudi Arabia. 'Buraidah Central Hospital, Buraidah, Saudi Arabia.

\section{Received: 18 October 2018 Accepted: 10 October 2019}

Published online: 22 October 2019

\section{References}

1. World Health Organization: Middle East Respiratory Syndrome Coronavirus (MERS-CoV) Fact Sheet. 2018.

2. Su S, Wong G, Shi W, Liu J, Lai ACK, Zhou J, Liu W, Bi Y, Gao GF Epidemiology, genetic recombination, and pathogenesis of coronaviruses. Trends Microbiol. 2016;24(6):490-502.

3. Mohd HA, Al-Tawfiq JA, Memish ZA. Middle East respiratory syndrome coronavirus (MERS-CoV) origin and animal reservoir. Virol J. 2016;13:87.

4. Wong G, Liu W, Liu Y, Zhou B, Bi Y, Gao GF. MERS, SARS, and Ebola: the role of super-spreaders in infectious disease. Cell Host Microbe. 2015;18(4):398-401.

5. Omrani AS, Matin MA, Haddad Q, Al-Nakhli D, Memish ZA, Albarrak AM. A family cluster of Middle East respiratory syndrome coronavirus infections related to a likely unrecognized asymptomatic or mild case. Int J Infect Dis. 2013;17(9):e668-72.

6. Khalid I, Alraddadi BM, Dairi Y, Khalid TJ, Kadri M, Alshukairi AN, Qushmaq IA. Acute management and long-term survival among subjects with severe Middle East respiratory syndrome coronavirus pneumonia and ARDS. Respir Care. 2016;61(3):340-8.

7. Du L, Yang Y, Zhou Y, Lu L, Li F, Jiang S. MERS-CoV spike protein: a key target for antivirals. Expert Opin Ther Targets. 2017:21(2):131-43.

8. Li Y, Wan Y, Liu P, Zhao J, Lu G, Qi J, Wang Q, Lu X, Wu Y, Liu W, et al. A humanized neutralizing antibody against MERS-CoV targeting the receptorbinding domain of the spike protein. Cell Res. 2015;25(11):1237-49.

9. Du L, Zhao G, Yang Y, Qiu H, Wang L, Kou Z, Tao X, Yu H, Sun S, Tseng CT, et al. A conformation-dependent neutralizing monoclonal antibody specifically targeting receptor-binding domain in Middle East respiratory syndrome coronavirus spike protein. J Virol. 2014;88(12):7045-53.

10. Raj VS, Smits SL, Provacia LB, van den Brand JM, Wiersma L, Ouwendijk WJ, Bestebroer TM, Spronken MI, van Amerongen G, Rottier PJ, et al. Adenosine deaminase acts as a natural antagonist for dipeptidyl peptidase 4-mediated entry of the Middle East respiratory syndrome coronavirus. J Virol. 2014;88(3):1834-8.

11. Qiu H, Sun S, Xiao H, Feng J, Guo Y, Tai W, Wang Y, Du L, Zhao G, Zhou Y. Single-dose treatment with a humanized neutralizing antibody affords full protection of a human transgenic mouse model from lethal Middle East respiratory syndrome (MERS)-coronavirus infection. Antivir Res. 2016;132:141-8.

12. Pascal KE, Coleman CM, Mujica AO, Kamat V, Badithe A, Fairhurst J, Hunt C, Strein J, Berrebi A, Sisk JM, et al. Pre- and postexposure efficacy of fully human antibodies against spike protein in a novel humanized mouse model of MERSCoV infection. Proc Natl Acad Sci U S A. 2015;112(28):8738-43.

13. Johnson RF, Bagci U, Keith L, Tang X, Mollura DJ, Zeitlin L, Qin J, Huzella L, Bartos CJ, Bohorova N, et al. 3B11-N, a monoclonal antibody against MERS$\mathrm{CoV}$, reduces lung pathology in rhesus monkeys following intratracheal inoculation of MERS-CoV Jordan-n3/2012. Virology. 2016;490:49-58.
14. Mo Y, Fisher D. A review of treatment modalities for Middle East respiratory syndrome. J Antimicrob Chemother. 2016;71(12):3340-50.

15. Al Ghamdi M, Alghamdi KM, Ghandoora Y, Alzahrani A, Salah F, Alsulami A, Bawayan MF, Vaidya D, Perl TM, Sood G. Treatment outcomes for patients with middle eastern respiratory syndrome coronavirus (MERS CoV) infection at a coronavirus referral center in the Kingdom of Saudi Arabia. BMC Infect Dis. 2016;16:174.

16. Arabi YM, Alothman A, Balkhy HH, Al-Dawood A, AlJohani S, Al Harbi S, Kojan S, Al Jeraisy M, Deeb AM, Assiri AM, et al. Treatment of Middle East respiratory syndrome with a combination of lopinavir-ritonavir and interferon-beta1b (MIRACLE trial): study protocol for a randomized controlled trial. Trials. 2018;19(1):81.

17. Modjarrad K. Treatment strategies for Middle East respiratory syndrome coronavirus. J Virus Erad. 2016;2(1):1-4.

18. Falzarano D, de Wit E, Rasmussen AL, Feldmann F, Okumura A, Scott DP, Brining D, Bushmaker T, Martellaro C, Baseler L, et al. Treatment with interferon-alpha2b and ribavirin improves outcome in MERS-CoV-infected rhesus macaques. Nat Med. 2013;19(10):1313-7.

19. Choi WS, Kang Cl, Kim Y, Choi JP, Joh JS, Shin HS, Kim G, Peck KR, Chung DR, Kim HO, et al. Clinical presentation and outcomes of Middle East respiratory syndrome in the Republic of Korea. Infect Chemother. 2016;48(2):118-26.

20. Al-Tawfiq JA, Momattin H, Dib J, Memish ZA. Ribavirin and interferon therapy in patients infected with the Middle East respiratory syndrome coronavirus: an observational study. Int J Infect Dis. 2014;20:42-6.

21. Almekhlafi GA, Albarrak MM, Mandourah Y, Hassan S, Alwan A, Abudayah A, Altayyar S, Mustafa M, Aldaghestani T, Alghamedi A, et al. Presentation and outcome of Middle East respiratory syndrome in Saudi intensive care unit patients. Crit Care. 2016;20(1):123.

22. Sherbini N, Iskandrani A, Kharaba A, Khalid G, Abduljawad M, Al-Jahdali H. Middle East respiratory syndrome coronavirus in Al-Madinah City, Saudi Arabia: demographic, clinical and survival data. J Epidemiol Glob Health. 2017;7(1):29-36.

23. Arabi YM, Arifi AA, Balkhy HH, Najm H, Aldawood AS, Ghabashi A, Hawa H, Alothman A, Khaldi A, Al Raiy B. Clinical course and outcomes of critically ill patients with Middle East respiratory syndrome coronavirus infection. Ann Intern Med. 2014;160(6):389-97.

24. Al-Hameed F, Wahla AS, Siddiqui S, Ghabashi A, Al-Shomrani M, Al-Thaqafi A, Tashkandi Y. Characteristics and outcomes of Middle East respiratory syndrome coronavirus patients admitted to an intensive care unit in Jeddah, Saudi Arabia. J Intensive Care Med. 2016;31(5):344-8.

25. Al-Tawfiq JA, Hinedi K, Ghandour J, Khairalla H, Musleh S, Ujayli A, Memish ZA. Middle East respiratory syndrome coronavirus: a case-control study of hospitalized patients. Clin Infect Dis. 2014;59(2):160-5.

26. Shalhoub S, Farahat F, Al-Jiffri A, Simhairi R, Shamma O, Siddiqi N, Mushtaq A. IFN-a2a or IFN-b1a in combination with ribavirin to treat middle Eastrespiratory syndrome coronavirus pneumonia: a retrospective study. J Antimicrob Chemother. 2015;70:2129-32.

27. Saad M, Omrani AS, Baig K, Bahloul A, Elzein F, Matin MA, Selim MA, Al Mutairi M, Al Nakhli D, Al Aidaroos AY, et al. Clinical aspects and outcomes of 70 patients with Middle East respiratory syndrome coronavirus infection: a single-center experience in Saudi Arabia. Int J Infect Dis. 2014;29:301-6.

28. Assiri A, Al-Tawfiq JA, Al-Rabeeah AA, Al-Rabiah FA, Al-Hajjar S, Al-Barrak A, Flemban H, Al-Nassir WN, Balkhy HH, Al-Hakeem RF, et al. Epidemiological, demographic, and clinical characteristics of 47 cases of Middle East respiratory syndrome coronavirus disease from Saudi Arabia: a descriptive study. Lancet Infect Dis. 2013;13(9):752-61.

29. Omrani AS, Saad MM, Baig K, Bahloul A, Abdul-Matin M, Alaidaroos AY, Almakhlafi GA, Albarrak MM, Memish ZA, Albarrak AM. Ribavirin and interferon alfa-2a for severe Middle East respiratory syndrome coronavirus infection: a retrospective cohort study. Lancet Infect Dis. 2014;14(11):1090-5.

30. Alraddadi B, Bawareth N, Omar H, Alsalmi H, Alshukairi A, Qushmaq I, Feteih M, Qutob M, Wali G, Khalid I. Patient characteristics infected with Middle East respiratory syndrome coronavirus infection in a tertiary hospital. Ann Thorac Med. 2016;11(2):128-31.

31. Al-Abdallat MM, Payne DC, Alqasrawi S, Rha B, Tohme RA, Abedi GR, Al Nsour M, Iblan I, Jarour N, Farag NH, et al. Hospital-associated outbreak of Middle East respiratory syndrome coronavirus: a serologic, epidemiologic, and clinical description. Clin Infect Dis. 2014;59(9):1225-33.

\section{Publisher's Note}

Springer Nature remains neutral with regard to jurisdictional claims in published maps and institutional affiliations. 